\section{Adaptation in distance perception based on oculomotor cues*}

\author{
HANS WALLACH and KARL JOSEF FREY \\ Swarthmore College, Swarthmore, Pennsylvania 19081
}

Accommodation and convergence primarily serve to adjust the eyes to the distance of the object viewed, but, once made, these oculomotor adjustments serve as cues for the object's distance. Experiments are reported that show that the relation between oculomotor adjustments and the distances they signify can be changed by adaptation to glasses that cause alteration in the oculomotor adjustments with which objects are viewed. This changed relation manifested itself in marked alterations of size perception. Wearing, for $30 \mathrm{~min}$, glasses that caused a change in accommodation and convergence corresponding to a smaller object distance and equivalent to 1.5 lens diopters caused subsequent mean size increases that ranged from $50 \%$ to $65 \%$. Adaptation to glasses that changed oculomotor adjustments in the same amount but in the opposite direction resulted in decreases in perceived sizes that varied from $18 \%$ to $40 \%$, dependent on the distance of the test object. These were the results of size estimates obtained before and after the adaptation period under conditions where only accommodation and convergence served as cues for distance. A newly developed test of size perception was also used, in which $S$ adjusted the size of the projected image of an array of familiar objects on a screen until the size of the objects appeared normal. Again, such adjustments were made before and after the adaptation period, and size differences were obtained that were in the direction to be expected of adaptation and varied in amount between $12 \%$ and $33 \%$, dependent on the distance of the screen. The reason for the different amounts of size change measured by the two kinds of tests was investigated.

The experiments by Wallach and Floor (1971) showed that accommodation and convergence in conjunction operate as fairly adquate cues for shorter distances of objects from $S$. This made feasible an attempt to change through adaptation the relationship between these oculomotor adjustments and the distances they denote. The spectacles we used for this purpose changed accommodation and convergence by corresponding amounts at all object distances. 1 There were actually two spectacles that had opposite effects. One consisted of -1.5-diopter spherical lenses and 5-diopter prisms with bases in temporal position. They caused the eyes to accommodate 1.5 diopters more than the object distance would warrant and converge by an additional 5 prism diopters. These are oculomotor adjustments that corresponded to a shorter than the true object distance. Hence, these spectacles will be called "near glasses." The "far glasses" consisted of +1.5-diopter lenses and 5-diopter prisms with base nasal, and forced the eyes into adjustments equivalent to a larger object distance. Inasmuch as the change in accommodation and the change in convergence caused by such glasses are always equivalent, ${ }^{2}$ all our

* This work was supported by Grant GB 5958 from the National Science Foundation to Swarthmore College, Hans Wallach, principal investigator. considerations will be conducted in terms of the forced lens adjustments, implying always a corresponding adjustment of convergence.

Two facts about the effect of these glasses need to be kept in mind. They do not change oculomotor adjustment proportionately but additively. By causing the eyes to change adjustment to a given object distance by the equivalent of 1.5 lens diopters, they force an increase in accommodation and convergence corresponding to this diopter value in the case of the near glasses and an identical decrease in the case of the far glasses. An increase in accommodation and convergence corresponds to a shorter distance of the point on which the eyes are focused and converged, and a decrease corresponds to a larger object distance. Besides, there is the general fact about the diopter measure that the viewing distance that corresponds to a certain oculomotor adjustment is the reciprocal in meters of its lens diopter equivalent. Thus, the dioptric change caused by the glasses corresponds to vastly different changes in viewing distance, dependent on the distance of the object. For instance, normal oculomotor adjustment to a distance of $1 \mathrm{~m}$ is equivalent to 1 lens diopter. With the near glasses in place, the adjustment amounts to 1.5 diopters more, i.e., 2.5 diopters, for the same object distance. An oculomotor adjustment amounting to 2.5 diopters, in turn, corresponds to a distance of
$40 \mathrm{~cm}$. An object at infinity, on the other hand, whose distance is equivalent to 0 diopters, will, through the glasses, be seen with an adjustment of 1.5 diopters, which corresponds to a distance of $66.7 \mathrm{~cm}$.

Henceforth, we shall call a distance that corresponds to an oculomotor adjustment forced by our glasses "equivalent distance."3 The near glasses do not only diminish the equivalent distances with which objects are viewed; they also contract the range of equivalent distances. Because the near glasses force the eyes to accommodate and converge an additional 1.5 lens diopters, oculomotor adjustment cannot be less than 1.5 diopters, and the largest equivalent distance possible is $66.7 \mathrm{~cm}$ when objective distances comprise the entire range. Since the far glasses diminish oculomotor adjustments to all object distances by 1.5 diopters and increase the equivalent distance with which objects are viewed, they do not limit the range of equivalent distances, which thus can vary from infinity to the near point of accommodation. But they severely limit the object distances to which the eyes equipped with these far glasses can adjust. Since they force the eyes to look at objects with an adjustment that is $\mathbf{1 . 5}$ diopters less than their objective distance would warrant, an object $66.7 \mathrm{~cm}$ distant will be viewed with an oculomotor adjustment for infinity. Thus, the largest possible object distance is $66.7 \mathrm{~cm}$. This limits the possible depth of space around an $S$ wearing the far glasses to $66.7 \mathrm{~cm}$.

What perceptual effects do such spectacles produce and what effect of adaptation can be expected from wearing them? These glasses alter the oculomotor adjustment with which objects are seen and hence their distances insofar as these distances depend on oculomotor adjustments. Among the several consequences of the altered distances, the following is the most important: Where distance cues other than accommodation and convergence are largely absent, the apparent sizes of objects should be altered, and this is indeed the case. Seen through the far glasses, objects appear larger than without them and through the near glasses, smaller. This is a consequence of the rule of size perception, that perceived size depends on the size of the retinal image of the object and the cues that are received for the object's distance. More accurately, perceived size is equivalent to image size times registered distance (Emmert's law), where registered distance is the representation of distance in the nervous system, usually the result of the various distance cues available. 
Thus, the alteration of oculomotor adjustment caused by the glasses amounts to an alteration of registered distance and a change in perceived size results. An adaptation to our glasses would mean that the relation between oculomotor adjustment and registered distance is so altered that the latter is in better agreement with object distance. With adaptation complete, registered distance based on oculomotor adjustment and hence size perception would become veridical when the glasses are worn. Thus, some measurement involving size perception, once before and once after the adaptation period, should measure the adaptation effect. 4

We measured adaptation with two such tests, both involving the role that registered distance plays in Emmert's law. In one of these tests, $S$ gave estimates of the apparent length of the diagonal of luminous diamond shapes seen in the dark, by selecting and by adjusting the length of small metal rods using the sense of touch only (size estimation test). These estimates represented perceived size, which was thus the dependent variable in this test. The other test utilized the fact that certain objects occur in standard sizes only. A color slide of such objects was projected on a screen, and
$S$ was asked to adjust the size of the projected images until the size of the objects shown appeared normal to him (normal size adjustment test). In this test, a different use was made of Emmert's law. In the case of an object of standard size, the perceived size is fixed and, at the time of the test, supplied by memory. The size of the retinal image is here the dependent variable. Varying this image size (by changing the size of the projected image) until the size of the object appears normal therefore measures registered distance. If $S$ adjusts the size of the image on the screen so that it is the true size of the object, registered distance agrees with the true distance of the screen. An adjustment of the projected image to smaller than normal means that registered distance is larger than the true distance, because a smaller size of the screen image and hence a smaller retinal image means, in the case of an object of normal size, that the object is farther.

The limitation of the equivalent distances inherent in the near glasses made it desirable to do the tests without them; the glasses were worn only during the period of adaptation. Thus, adaptation was not measured as a decrease in the change that viewing objects through the glasses causes, but as an error in natural vision. After adaptation to the near glasses, when registered distances were expected to be enlarged, estimated sizes would be, by Emmert's law, larger than they ordinarily are and adjustments to normal size, smaller, since adaptation to near glasses causes changes in the direction of those produced by wearing far glasses. For the far glasses, the opposite effects were expected. The mentioned limitations also prompted us to place the test displays at various distances from $\mathrm{S}$. In the size estimation test, figures were presented at $25,50,100$, and $200 \mathrm{~cm}$ from $\mathrm{S}$, and in the normal size adjustment test the screen distances were $25,50,100$, and $150 \mathrm{~cm}$. In the case of the near glasses, the two larger test distances were outside the range of equivalent distances possible when the glasses are worn, since then oculomotor adjustments of less than 1.5 diopters cannot occur. So the question arose as to whether an adaptation acquired with oculomotor adjustments of more than 1.5 diopters could manifest itself at distances corresponding to less than 1.5 diopters, that is, at the two larger of the four test distances. This question did not arise for the far glasses where the whole range of
SIZE

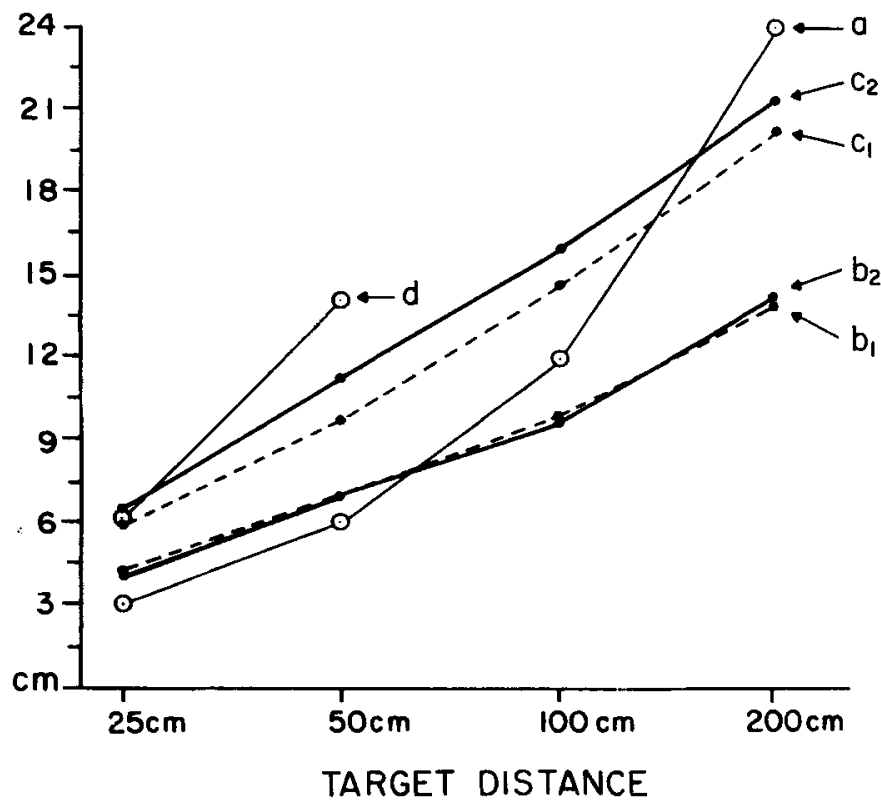

Fig. 1. Mean size estimates made before and after adaptation to near glasses. Curve a represents the objective target sizes. In Curves $b_{1}$ and $b_{2}$ the means of the preadaptation size estimates made in two experiments are given. Curve $c_{1}$ shows the mean size estimates after $15 \mathrm{~min}$ of adaptation and Curve $c_{2}$ after $30 \mathrm{~min}$ of adaptation. Curve $d$ gives the sizes predicted for complete adaptation, based on preadaptation size estimates taken at the distances representing complete adaptation.

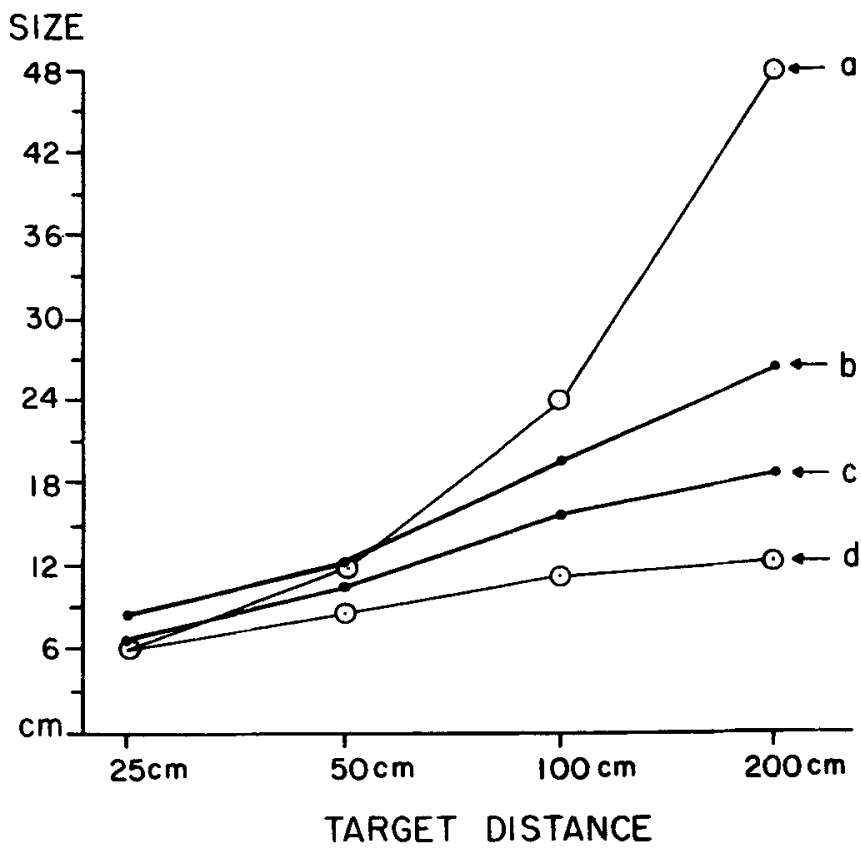

Fig. 2. Mean size estimates made before and after adaptation to far glasses. Curve a represents the objective target sizes. In Curve $b$ the means of the preadaptation size estimates are given. Curve $c$ shows the mean size estimates after $\mathbf{3 0}$ min of adaptation. Curve $d$ gives the sizes predicted for complete adaptation, based on preadaptation size estimates taken at the distances representing complete adaptation. 
Table 1

Adaptation to Neax Glasses With 15 Min of Block Moving (A) and With $15 \mathrm{Min}$ of Block Moving Plus $15 \mathrm{Min}$ of Walking (B) Measured (in Centimeters) With the Size Estimation Test. $\mathbf{N}=24$

\begin{tabular}{|c|c|c|c|c|c|c|}
\hline 1 & Test distance & & 25 & 50 & 100 & 200 \\
\hline 2 & Target size & & 3 & 6 & 12 & $\mathbf{2 4}$ \\
\hline $\mathbf{3}$ & $\begin{array}{l}\text { Preadaptation } \\
\text { size estimate }\end{array}$ & $\begin{array}{l}\mathbf{A} \\
\mathbf{B}\end{array}$ & $\begin{array}{l}4.19 \\
4.14\end{array}$ & $\begin{array}{l}6.98 \\
7.00\end{array}$ & $\begin{array}{l}9.92 \\
9.65\end{array}$ & $\begin{array}{l}13.98 \\
14.30\end{array}$ \\
\hline 4 & $\begin{array}{l}\text { Postadaptation } \\
\text { size estimate }\end{array}$ & $\begin{array}{l}\mathbf{A} \\
\mathbf{B}\end{array}$ & $\begin{array}{l}5.91 \\
6.45\end{array}$ & $\begin{array}{r}9.82 \\
11.28\end{array}$ & $\begin{array}{l}14.70 \\
16.01\end{array}$ & $\begin{array}{l}20.30 \\
21.46\end{array}$ \\
\hline 5 & $\begin{array}{l}\text { Difference between pre- and } \\
\text { postadaptation size estimates }\end{array}$ & $\begin{array}{l}\mathbf{A} \\
\mathbf{B}\end{array}$ & $\begin{array}{l}1.72 \\
2.31\end{array}$ & $\begin{array}{l}2.84 \\
4.28\end{array}$ & $\begin{array}{l}4.78 \\
6.36\end{array}$ & $\begin{array}{l}6.32 \\
7.16\end{array}$ \\
\hline 6 & $\begin{array}{l}\text { Adaptation effect } \\
\text { as size increase }\end{array}$ & $\begin{array}{l}\mathbf{A} \\
\mathbf{B}\end{array}$ & $\begin{array}{l}41.81 \% \\
58.07 \%\end{array}$ & $\begin{array}{l}41.24 \% \\
61.79 \%\end{array}$ & $\begin{array}{l}47.63 \% \\
65.34 \%\end{array}$ & $\begin{array}{l}43.15 \% \\
50.62 \%\end{array}$ \\
\hline
\end{tabular}

oculomotor adjustments could occur during adaptation.

Where adaptation is to a device that alters cues so that they misrepresent objective conditions, as is the case with our glasses, adaptation consists in a modification of the perceptual process evoked by these cues such that veridicality of perception is partly or completely restored. However, such a modification obviously can take place only when other cues are given that represent the true objective condition. In the case of the cues affected by our glasses, namely, oculomotor adjustment, there are several cues and conditions that can do this, such as perspective cues for distance, cues for distance produced by S's locomotion and his manipulations, and perhaps the image sizes of familiar objects. Since we wanted to obtain large adaptation effects, we tried to make as many of these cues available in the adaptation period as was feasible. Because of the limitation of object distances to $66.7 \mathrm{~cm}$ required by the far glasses, two different conditions for adaptation were used in the case of the near glasses, one that was easily altered to conform to the limitations set by the far glasses (Condition A) and another one that promised optimal conditions for adaptation (Condition B). Since we aimed at as strong an adaptation effect as compatible with adaptation periods of feasible length, $15 \mathrm{~min}$ for each, we gave $S$ Condition $A$, tested for adaptation, and continued immediately with Adaptation Condition B, followed by another postadaptation test. In this fashion, the effect of Condition $B$ was added to what adaptation was left at the end of the test for Condition $A$. In the case of the near glasses, two experimental sessions were run, one in which the normal size adjustment (NSA) test followed Condition $A$ and the size estimation (SE) test followed Condition $B$, and another where the test order was reversed. The sequence of adaptation conditions was not varied. Only one experimental session was run with the far glasses. Here the NSA test always followed the first adaptation period and the SE test, the second.

\section{APPARATUS AND PROCEDURE The Adaptation Periods}

Our spectacles consisted of one meniscus lens for each eye, ground to provide both the spherical lens action of \pm 1.5 diopters and the prism action of 5 diopters. The lenses were $49 \mathrm{~mm}$ in diam and fitted into welder's goggles where they replaced the glass filters. There was room inside the goggles to accommodate ordinary eyeglasses, and Ss who needed minor corrections could thus participate in our experiments. We only excluded Ss who wore strong corrective glasses.

The adaptation periods for the near glasses were as follows: In Condition $A, S$ sat at a table covered by a white cardboard panel, on a stool $51 \mathrm{~cm}$ high. His task was to move by hand or with a small rake 10 differently shaped wooden blocks back and forth across the table. To make this task a bit more interesting, one or another panel marked with the 10 outlines of the bases of these blocks covered the table and $S$ had to fit each block into the outline of its base. In Condition B, $S$ walked for $15 \mathrm{~min}$ a long, narrow hallway that provided clear perspective depth cues. Four stands on which white cardboard squares, $30 \mathrm{~cm}$ large and mounted approximately at eye level, were evenly spaced along the path of $S$, who would naturally look at each square as he approached it. E accompanied $S$ on this walk.

In the case of the far glasses, Condition A was as described above, except for the discussed spatial limitations. A large cardboard box of cubic shape and $80 \mathrm{~cm}$ in size was placed on the table so that its far wall was $60 \mathrm{~cm}$ from the edge at which $S$ sat. This enclosure, the inside of which was papered with richly patterned material, was so placed around $S$ that his view was in all directions restricted by walls whose distance nowhere exceeded $65 \mathrm{~cm}$. In Condition B, S sat in the same enclosure and worked for $15 \mathrm{~min}$ on wooden block puzzles.

\section{The Size Estimation Test}

As mentioned, the diamond-shaped test objects were presented at four distances. Their sizes were chosen so that they produced identical retinal images. At the distances of 25, 50, 100 , and $200 \mathrm{~cm}$, their diagonals were $3,6,12$, and $24 \mathrm{~cm}$, respectively, when adaptation was to the near glasses. In that case, an increase in the apparent size was expected after the adaptation period. Because adaptation to the far glasses would manifest itself in a decrease in apparent size, larger test objects were used; they measured $6,12,24$, and $48 \mathrm{~cm}$ along the diagonal. A lightbox on wheels and movable on a track was used to present the various test objects by means of a diamond-shaped aperture of variable size (Aubert diaphragm) over a milk-glass face. To allow $\mathbf{S}$ to move his head during the lengthy test period, we assured the proper location of S's head while the test object was visible in the following manner: $\mathbf{S}$ wore a headgear that could be attached to a vertical shaft which turned in a bearing mounted above S's seat. When S's head was so positioned that its median plane was intersecting the center of the test object, the shaft was held in position by a stop and simultaneously operated a microswitch that caused the test object to light up; when the head was in any other position, the test object was not visible. This arrangement prevented movement parallax from operating as a distance cue.

Black drapes on either side of the track and a screen in front of the lightbox prevented $\mathbf{S}$ from seeing any part of the test arrangement while the room was lit. Only after S's head was attached to the shaft and the room had been darkened was the screen lowered to the point where $S$ could see the whole diamond shape, but none of the light reflected by the tracks beneath it. In short, all known distance cues other than accommodation and convergence were eliminated from the test conditions. Had any been operating, they would have provided veridical information about the distances of the test objects and would have counteracted the adaptation effect in the postadaptation test.

There were 12 metal rods which $S$ used to give his size estimate. Each one could be extended so that its length was nearly doubled or quadrupled. They measured from 1.5 to $12.7 \mathrm{~cm}$ in their unextended state. Size estimates 
using only the sense of touch were required, because had the rods been visible the changed size perception caused by adaptation would also have affected the apparent size of the rods in the postadaptation tests. Single estimates were given for each of the four test diamonds at the four test distances, one before and the other after the adaptation period, with the order of presentation varied from $S$ to $S$. For a given $S$, the test order was the same in the pre- and postadaptation tests.

Since, in the tests, only accommodation and convergence were available as distance cues, the preadaptation size estimates fall considerably short of constancy. A comparison of Curve $b$, representing preadaptation size estimates, with Curve $a$, representing object sizes in Fig. 1 or Fig. 2, shows how greatly the sizes of the more distant test objects were underestimated. This fact becomes important when we want to compare the adaptation effects that were actually obtained with the size estimates to be obtained under the assumption that adaptation was complete. The theoretical distance of a test object under the assumption of complete adaptation is easily computed. The near glasses, for instance, force the eyes into an oculomotor adjustment that is $\mathbf{1 . 5}$ lens diopters greater than the object distance would require. Complete adaptation to this condition involves compensation for the whole amount by which the equivalent distance is shorter than the true object distances and means that the registered distance that corresponds to such an oculomotor adjustment is larger by the equivalent of 1.5 diopters than it would normally be. Thus, to compute the theoretical adaptation distance that corresponds to a given test distance, we subtract $\mathbf{1 . 5}$ diopters from the diopter equivalent of that test distance and reconvert the result into distance. While the theoretical distance corresponding to complete adaptation is thus easily derived, a theoretical postadaptation size estimate expected to result from the theoretical adaptation distance must be empirically determined. We therefore obtained, prior to adaptation, size estimates at the theoretical adaptation distances that correspond to our test distances. This we did immediately after the preadaptation size estimates at the four normal test distances had been obtained. In the case of the far glasses, the theoretical adaptation distances were $18.2,28.6,40$, and $50 \mathrm{~cm}$ for the test distances of $25,50,100$, and $200 \mathrm{~cm}$, respectively. In the case of the near glasses, only two of the theoretical adaptation distances were meaningful, namely, $40 \mathrm{~cm}$ for the test distance of $25 \mathrm{~cm}$ and $200 \mathrm{~cm}$ for the distance of $50 \mathrm{~cm}$. For the larger test distances, theoretical adaptation distances were at infinity. Curves $d$ in Figs. 1 and 2 give the size estimates produced at the theoretical adaptation distances. The estimates are represented in the graphs not at the distances at which they were actually measured, but at the main test distances to which they refer.

\section{The Normal Size \\ Adjustment Test}

A color slide was made of five standard-sized objects: a 12-in. ruler, a BIC pen, a dollar bill, a matchbook, and a wrapped piece of chewing gum. A Kodak Carousel 800 projector equipped with a 3-in. lens projected it from the rear on a translucent plastic screen, $70 \mathrm{~cm}$ wide and $60 \mathrm{~cm}$ high. Projector and screen were mounted on separate four-wheel carriages, both of which rode on a 4-m-long track. At one end of the track, an adjustable chinrest and a horizontal bar above it, against which $S$ leaned his forehead, located S's eyes at the height of the projector lens and thus of the center of the slide image. The size of the projected image could be varied by changing the distance between the projector and the screen. A black metal disk with a concentric opening $25 \mathrm{~mm}$ in diam was fitted into the front end of the projector lens to insure a sharp image at all projection distances. $S$ controlled the size of the projected image by turning a crank which, by means of a looped cord and a system of pulleys, caused the projector carriage to move toward or away from the screen. Every effort was made to eliminate all distance cues other than oculomotor adjustments from the test conditions. There was one flaw: internal reflections in the projector lens produced a faint glow of the whole screen which, thus, became an object of fixed size at all test distances.

For each screen distance, S made two normal size adjustments, one where $\mathbf{E}$ had placed the projector so that the size of the image on the screen was clearly too large, while for the other the initial image size was made to look too small. The average of these two settings, whose order was randomized, constituted an individual score. Again, the order of the four screen distances was varied from $S$ to $\mathbf{S}$. For a given $\mathbf{S}$, the same order was used in the pre- and postadaptation adjustment, and it was identical with the order employed in the SE test.

\section{Subjects}

The same Ss were used in all three experiments, the two versions of adaptation to the near glasses and one concerned with adaptation to the far glasses. The time interval between two experiments was at least 3 weeks, and adaptation to the far glasses preceded the second near-glasses version. Ss, paid undergraduates, were selected for good distance perception based on oculomotor adjustment. To be employed, an $\mathbf{S}$ had to give, in the preadaptation $S E$ test, four size estimates that increased with increasing size of the diamond shape, and in the first NSA test the adjustment produced for the $150-\mathrm{cm}$ screen distance could not exceed the true size of the objects by a factor larger than 1.5. To select our $24 \mathrm{Ss}$, a total of 48 had to be given the preadaptation tests.

\section{RESULTS}

Large and highly significant adaptation effects were obtained with these selected Ss. In the case of the SE test, which yielded the strongest effects, not a single postadaptation score failed to differ from the corresponding preadaptation score in the direction of adaptation, and the same was true of the NSA test results for the near glasses-a total of 432 individual scores.

The Size Estimation Test

Results of the near glasses. It will be remembered that adaptation to the near glasses was twice performed with the same group of 24 Ss. In one experiment the $\mathrm{SE}$ test followed Adaptation Condition $A$, which always preceded Condition $B$, and in the other it followed Condition B. The SE results for both experiments are presented in Table 1 . Since the two experiments were done some time apart, the preadaptation estimates

Table 2

Adaptation to Far Glasses With 15 Min of Block Moving and 15 Min of Doing Wooden Puzzles Measured (in Centimeters) With the Size Estimation Test. $(N=12)$

\begin{tabular}{|c|c|c|c|c|c|}
\hline $\mathbf{1}$ & Test distance & $\mathbf{2 5}$ & 50 & 100 & 200 \\
\hline 2 & Target size & 6 & 12 & 24 & 48 \\
\hline 3 & Preadaptation size estimate & 8.43 & 12.39 & 19.71 & 26.51 \\
\hline 4 & Postadaptation size estimate & 6.73 & 10.43 & 15.77 & 18.80 \\
\hline 5 & $\begin{array}{l}\text { Difference between pre- and } \\
\text { postadaptation size estimates }\end{array}$ & 1.70 & 1.96 & 3.94 & 7.71 \\
\hline 6 & Adaptation effect & $\mathbf{2 7 . 1 1 \%}$ & $18.70 \%$ & $27.44 \%$ & $40.43 \%$ \\
\hline
\end{tabular}


Table $\mathbf{3}$

Adaptation to Near Glasses With 15 Min of Block Moving (A) and With 15 Min of Block Moving Plus 15 Min of Walking (B) Measured With the Normal Size Adjustment Test. $N=24$

1 Screen distance

Preadaptation normal size adjust-

2 ment in percent of true normal size

Postadaptation normal size adjust-

3 ment in percent of true normal size

Mean ratio of preadaptation score over postadaptation score as size decrease
- Adaptation effect

\begin{tabular}{lrrr}
$25 \mathrm{~cm}$ & $50 \mathrm{~cm}$ & $100 \mathrm{~cm}$ & $150 \mathrm{~cm}$ \\
$98.40 \%$ & $100.92 \%$ & $102.71 \%$ & $111.09 \%$ \\
$93.90 \%$ & $96.89 \%$ & $99.98 \%$ & $104.63 \%$ \\
$88.62 \%$ & $87.93 \%$ & $84.31 \%$ & $91.63 \%$ \\
$83.88 \%$ & $79.94 \%$ & $76.08 \%$ & $81.20 \%$ \\
1.1128 & 1.1537 & 1.2229 & 1.2239 \\
1.1220 & 1.2202 & 1.3351 & 1.3075 \\
$11.28 \%$ & $15.37 \%$ & $22.29 \%$ & $22.39 \%$ \\
$12.20 \%$ & $22.02 \%$ & $33.51 \%$ & $30.75 \%$ \\
\hline
\end{tabular}

were taken twice also. The close agreement between the means of the two sets of scores presented in Lines $3 \mathrm{~A}$ and $3 \mathrm{~B}$ demonstrates the repeatability of these measurements. The difference between the preadaptation and the postadaptation size estimates (Line 5) represents, of course, the adaptation effect. The means of these difference scores are given as percent size increase in Line 6.

The means given in Line 5 of Table 1 show that the differences between the pre- and postadaptation size estimates increased with greater test distances. But this was merely due to the fact that the objective sizes of the test diamond increased in proportion to the test distance. Expressed as proportional size increase (Line 6), the effect of adaptation on size was approximately the same for all distances. Analyses of variance on the means of Lines $6 \mathrm{~A}$ and $6 \mathrm{~B}$ yielded no significance as a function of the distance of the test object $(F=.41$ and $F=1.38$, respectively). There was a difference in the mean effects on size provided by Condition $A$ and Condition $\mathrm{B}$ (compare Lines 6A and $6 B$ ), with the latter yielding a significantly larger adaptation effect $(F=15.38 ; \quad p<.005)$. Our data provide no means for determining whether the larger effect measured after Condition $B$ was due to the greater effectiveness of Adaptation Condition B as such or due to a cumulative effect of the two adaptation periods.

That an effect of adaptation was measured at the two larger test distances represents a transfer of adaptation to oculomotor adjustments that never occurred during the wearing of the near glasses. But the fact that the adaptation effects expressed as proportional size increases are approximately the same at all test distances does not necessarily mean that this transfer of the adaptation effect to the "unpracticed" oculomotor adjustments was complete. Inasmuch as our glasses force the eyes to change oculomotor adjustments by the equivalent of
1.5 diopters at all object distances (while the changes in distance that correspond to these changes in oculomotor adjustment vary widely with object distance), it seems plausible that adaptation, that is, the change of the registered distance that belongs to a given oculomotor adjustment, should also be of constant diopter value at all object distances. To find out whether this was the case, we transformed the size changes due to adaptation into their diopter equivalents. The size changes were transformed into distance changes by a method that took the underestimation of the sizes of the more distant test objects into account. ${ }^{5}$ The diopter equivalents of these distance changes were found to decrease strongly with increasing test distances. At the test distances of $25,50,100$, and $200 \mathrm{~cm}$, the dioptric changes for Condition $A$ amounted to $1.44,1.00, .53$, and .30 , respectively. To be sure, adaptation effects larger than 1 and .5 diopters cannot be expected for the test distances of 100 and $200 \mathrm{~cm}$, respectively, because such changes would correspond to objects at infinity. But the obtained values of .53 and .30 diopters are considerably smaller than these limitations, and a diminished transfer of the adaptation effect to oculomotor adjustments that did not occur during the adaptation period is a possibility.

Results for the far glasses. These results are listed in Table 2 , whose arrangement is identical with Table 1.6 Here the SE test was given only once, at the end of the second adaptation period. A comparison of Line 6 in Table 2 with Line 6 B in Table 1 shows that the proportional size changes due to adaptation to the far glasses-here the changes were, of course, decreases-were not as homogeneous as those to the near glasses; the effect at the $50-\mathrm{cm}$ test distance was, in fact, significantly smaller than that at the 200-cm distance. On the whole, the size effects obtained with the far glasses were significantly smaller than those due to the near glasses $(F=22.67, p<.005)$, but this result is probably immaterial. It can be ascribed to the fact that in the case of the far glasses adaptation caused registered distances to be diminished rather than enlarged, and identical dioptric changes would be expected to correspond to smaller distance and hence size changes.

The Normal Size Adjustment Test

The results for the NSA tests are presented in Tables 3 and 4 . Here the mean pre- and postadaptation scores (Lines 2 and 3 ) are given as ratios of the size adjustments that $S$ had made over the true sizes of the objects represented, multiplied by 100 . Thus, a $100 \%$ entry would mean that $S$ had succeeded in adjusting the image size on the screen to the true size of the object. As the preadaptation values in Line 2 show, surprisingly accurate adjustment means were obtained at all four test distances. In the case of near glasses (Table 3) the postadaptation adjustments are diminished in size, and this is in agreement with an increase in registered distance which adaptation to these glasses consists in. Under the assumption of complete constancy, the product of a retinal image size and registered distance is, for an object of standard size, a constant. Hence, when the registered distance increases as it should, the retinal image size should decrease. Since the objective distance of the screen remains unaltered, the size of the screen image must be made smaller to produce a smaller retinal image.

In Line 4 , the mean ratios of preadaptation adjustments over postadaptation adjustments are given, and in Line 5, the mean adaptation effects are listed as percentage size decreases; the latter, then, corresponds to Line 6 in Table 1 . As was the case with the results obtained with the $S E$ test, the size changes were significantly greater for Condition $B$ than for Condition A $(F=18.79 ; p<.005)$. But the results differed from those of the SE test in that the size changes increased significantly with test distance $(F=18.99$ and 32.54 ; $p<.005$ for each).

The results for the far glasses are presented in Table 4. Here adaptation provided an increase in size adjustments because it caused smaller registered distances, which, according to Emmert's law, require larger image sizes on the retina and on the screen in order to produce the same (normal) perceived sizes. Though small, the adaptation effects were quite significant. They serve to demonstrate that significant adaptation effects in both directions can be obtained with the NSA test also. 
The Difference Between the SE Test and the NSA Test Results

A comparison of Table 1 with Table 3 shows a striking difference in the amount of adaptation measured with the two tests. The size effects measured with the SE test were about 2.7 times larger. This difference was highly significant for both conditions, $A$ and $B(F=54.8$ and 44.3). When each test distance was separately considered, Duncan's new multiple range test showed that each difference between the two tests was significant at the .005 level.

This was, however, not the only difference between the results of the two tests. The means of the preadaptation scores obtained with the NSA test (Table 3, Line 2) show much better size constancy than those of the SE test (Table 1, Line 3). When the means for the $25-\mathrm{cm}$ and the $100-\mathrm{cm}$ test distances were compared and constancy quotients were formed for the two types of tests, a quotient of .6 was found for the SE test and one of .96 for the NSA test, where a quotient of 1.0 would stand for complete constancy. We ascribe this much more correct size perception in the NSA test partly to the fact that the images of the objects on the screen provide fine lines, absent in the test diamonds, that serve as more distinct marks for accommodation and convergence. We demonstrated this fact by collecting size estimates using the SE test method and the projection equipment of the NSA test for two kinds of test objects: (1) the projected images of a dollar bill, one $25 \%$ smaller and the other $25 \%$ larger than normal, or (2) the projections of a luminous diamond duplicating the bill's image sizes. Two screen distances, 75 and $150 \mathrm{~cm}$, were used. Averaged over the two sizes, mean size estimates at these distances ran $12.6 \%$ and $21.1 \%$ larger for the bill images than for the diamonds $(p<.01)$. Moreover, the loss in constancy from the $75-\mathrm{cm}$ distance to the $150-\mathrm{cm}$ distance was significantly larger for the diamond than for the bill $(p<.01)$.

The other fact accounting for the better size perception in the NSA test is the presence of extraneous distance

Table 5

Mean Size Estimates and Mean Size Adjustments Made With and Without Glasses Compared Size Estimation Normal Size Adjustment

Actual and

Equivalent Distances \&t

\begin{tabular}{|c|c|c|c|c|c|}
\hline \multirow{2}{*}{\multicolumn{2}{|c|}{$\begin{array}{c}\text { Actual and } \\
\text { Equivalent Distances at }\end{array}$}} & \multirow[b]{2}{*}{$33.3 \mathrm{~cm}$} & \multirow[b]{2}{*}{$66.7 \mathrm{~cm}$} & \\
\hline & & & & $33.3 \mathrm{~cm}$ & $66.7 \mathrm{~cm}$ \\
\hline $\mathbf{1}$ & Actual & 8.02 & 12.76 & .9548 & .9917 \\
\hline 2 & Equivalent & 8.03 & 12.17 & 1.5607 & .5959 \\
\hline 3 & Corrected Equivalent & & & .7804 & 1.1918 \\
\hline 4 & Ratio & 1.0 & 1.048 & 1.223 & 1.202 \\
\hline
\end{tabular}

$66.7 \mathrm{~cm}$

$33.3 \mathrm{~cm}$

$66.7 \mathrm{~cm}$

cues, probably provided by the faint glow on the screen mentioned above. We demonstrated the effect of extraneous distance cues in the NSA setup by obtaining size estimates and normal size adjustments under three viewing conditions: without glasses, with near glasses, and with far glasses. The test distances under these three conditions were so chosen that the test distances where no glasses were worn were the same as the equivalent test distances based upon the action of the spectacles. We selected two actual test distances, $33.3 \mathrm{~cm}$ and $66.7 \mathrm{~cm}$, because they were 1.5 diopters apart. Therefore, a test display $66.7 \mathrm{~cm}$ distant would be seen through the near glasses with an oculomotor adjustment for $33.3 \mathrm{~cm}$ distance, while a display actually $33.3 \mathrm{~cm}$ away and seen through the far glasses would be at an equivalent distance of $66.7 \mathrm{~cm}$. By comparing size estimates and size adjustments made under these two conditions with measurements taken without glasses at these display distances, the effectiveness of the glasses could be assessed. Since extraneous distance cues are veridical and thus interfere with the effect of the glasses, which, of course, alter only oculomotor cues, their presence would cause registered distances to deviate from equivalent distances, and size judgments obtained with and without glasses at equivalent and actual distances should reflect this. If, on the other hand, only oculomotor cues were present in the test situation, then the results of tests taken with the glasses should be predictable from tests without glasses, where the actual distances of the displays were the same as the equivalent distances with glasses. This prediction is simple in the

Table 4

Adaptation to Far Glasses With $15 \mathrm{Min}$ of Block Moving Measured With the Normal Size Adjustment Test. $N=12$

\begin{tabular}{llcccc}
\hline 1 & Screen distance & $25 \mathrm{~cm}$ & $50 \mathrm{~cm}$ & $100 \mathrm{~cm}$ & $150 \mathrm{~cm}$ \\
2 & $\begin{array}{l}\text { Preadaptation normal size adjust- } \\
\text { ment in percent of true normal size }\end{array}$ & $93.20 \%$ & $93.23 \%$ & $97.34 \%$ & $97.74 \%$ \\
3 & $\begin{array}{l}\text { Postadaptation normal size adjust- } \\
\text { ment in percent of true normal size }\end{array}$ & $96.21 \%$ & $98.31 \%$ & $103.33 \%$ & $104.61 \%$ \\
4 & $\begin{array}{l}\text { Mean ratio of preadaptation } \\
\text { score over postadaptation score }\end{array}$ & 1.0323 & 1.0516 & 1.0592 & 1.0683 \\
5 & Adaptation effect as size increase & $3.23 \%$ & $5.16 \%$ & $5.92 \%$ & $6.83 \%$ \\
\hline
\end{tabular}

case of the SE test because, as before, we chose the sizes of the test objects so that they produced equal retinal images: $6 \mathrm{~cm}$ for the diamond at $33.3 \mathrm{~cm}$ and $12 \mathrm{~cm}$ for the one $66.7 \mathrm{~cm}$ distant. Agreement of registered distance with equivalent distance should therefore cause identical size estimates where equivalent distance and actual distance are the same. As SE test data in Table 5, Lines 1 and 2 , show, this was true of the two mean estimates obtained at the $33.3-\mathrm{cm}$ distance and nearly so for those at the $66.7-\mathrm{cm}$ distance. (The small difference between the mean estimates listed for this distance was not significant.) It is obvious that we had succeeded in excluding extraneous distance cues from the $S E$ test conditions.

In the case of the NSA test, the assumption of an agreement of registered distance with equivalent distance leads to a different prediction about the measured sizes. For a pair of distances, one equivalent and the other actual, this assumption predicts normal size adjustments that correspond to equal retinal image sizes. But since for such a pair the screen distances are different, corrections have to be made in the means of the adjusted sizes to make them comparable. In the case of the pair concerned with the far glasses, the adjustment without the glasses was made with a screen distance of $66.7 \mathrm{~cm}$, while the equivalent distance was produced by a screen image at $33.3 \mathrm{~cm}$ seen through the glasses. A normal size adjustment made at the latter distance would have to be half as large as one made at the equivalent distance of $66.7 \mathrm{~cm}$ in order for the two to produce the same retinal image size. To correct for this, a measured size adjustment made with the far glasses had to be doubled before it was compared with a size adjustment made at the actual distance. In the case of the near glasses, the actual distance was $33.3 \mathrm{~cm}$, and the equivalent distance was produced when the glasses were worn and the screen distance was $66.7 \mathrm{~cm}$. A normal size adjustment made under the latter condition, then, had to be halved to be comparable with one made at the actual distance of $33.3 \mathrm{~cm}$. The mean 
size adjustment ratios obtained under the actual and equivalent conditions are given also in Lines 1 and 2 of Table 5, and the corrected values for the equivalent condition in Line 3. A comparison between the values in Lines 1 and 3 shows a considerable discrepancy, significant at the .01 level of confidence, for the near glasses as well as for the far glasses. To obtain percentage values for this discrepancy, ratios were formed between the two mean normal size adjustments obtained at each pair of test distances (using the correction) in such a manner that the influence of extraneous distance cues caused them to be larger than one (Line 4). These ratios show that adjustments made with glasses deviate on the average by $20 \%$ or more from the adjustments made without glasses at the corresponding distances. This is a considerable effect and, together with the effect of the more distinct marks present in the NSA test display earlier reported, accounts for the much more correct size perception under the conditions of the NSA test.

\section{DISCUSSION}

The presence of extraneous distance cues, in turn, furnishes an explanation for the smaller adaptation effects measured with the NSA test. Because extraneous distance cues are veridical, they would be in conflict with the altered registered distances based on oculomotor cues and caused by adaptation. The extraneous cues could, therefore, be expected to inhibit the manifestation of the adaptive modification that the processes connected with oculomotor adjustment had undergone. The presence of extraneous distance cues made the NSA test a less valuable tool in the investigation of our adaptation, but it provided us with an interesting result of a different kind: Our adaptation can become manifest in the presence of extraneous and, hence, conflicting distance cues.

Throughout this presentation, one basic assumption has not been questioned, namely, that changes in perceived size due to adaptation result from changes in registered distance. While this assumption is plausible, it need be specifically tested. Experiments to do this have by now been completed and have confirmed this assumption. They will be reported in connection with an investigation where the nature of the veridical distance cues present in the adaptation conditions was systematically varied.

\section{REFERENCES}

LEIBOWITZ, H., \& MOORE, D. Role of changes in accommodation and convergence in the perception of size. Journal of the Optical Society of America, 1966, 56, 1120-1123.

WALLACH, H., \& FLOOR, L. The use of size matching to demonstrate the effectiveness of accommodation and convergence as cues for distance.
Perception \& Psychophysics, 1971, 10, 423-428. NOTES

1. Optical arrangements that changed accommodation and convergence in equivalent amounts were previously used by Leibowitz and Moore (1966).

2. This is due to the fact that both accommodation and convergence are inversely proportional to viewing distance. Five prism diopters correspond to 1.5 lens diopters accurately only for Ss whose interocular distance is $6.67 \mathrm{~cm}$ approximately the mean of our $s$ population.

3. This term was introduced by Leibowitz and Moore (1966).

4. One would expect that the altered oculomotor adjustment caused by the glasses would result in changed perceived distance, but such an effect is not clearly obtained. An object seen through the far glasses, though it looks larger, will not also look farther away. We ascribe this to an effect of perceived size on perceived distance. When it is seen through the far glasses, an object's equivalent distance is larger than its true distance, but the effect of this is counteracted by its increased perceived size. At any rate, we concluded that perceived distance was not a good indicator of registered distance and did not use estimates of distance to measure adaptation.

5. We derived the registered distances that corresponded to the postadaptation size estimates from a curve where the preadaptation size estimates were plotted against test object distances. The values of the postadaptation size estimates were found on this curve, and the corresponding distance values were read.

6. While in Table 1, Line 6, the adaptation effect on size is given as the ratio of mean postadaptation estimate over mean preadaptation estimate minus one, Line 6 in Table 2 gives mean preadaption over mean postadaptation estimate minus one.

(Accepted for publication May 14, 1971.) 